\title{
Efficacy and Tolerability of Telmisartan Plus Amlodipine in Asian Patients Not Adequately Controlled on Either Monotherapy or on Low-Dose Combination Therapy
}

\author{
Dingliang Zhu, ${ }^{1}$ Pingjin Gao, ${ }^{1}$ Nobutaka Yagi, ${ }^{2}$ and Helmut Schumacher ${ }^{3}$ \\ ${ }^{1}$ Ruijin Hospital, Shanghai Jiaotong University School of Medicine, Shanghai Institute of Hypertension, \\ 197 Ruijin Road, Shanghai 200025, China \\ ${ }^{2}$ Nippon Boehringer Ingelheim Co., Ltd, 2-1-1, Osaki, Shinagawa-ku, Tokyo 141-6017, Japan \\ ${ }^{3}$ Boehringer Ingelheim Pharma GmbH \& Co. KG, Binger Straße 173, 55216 Ingelheim, Germany \\ Correspondence should be addressed to Dingliang Zhu; zhudingliang@sibs.ac.cn
}

Received 6 November 2013; Accepted 15 January 2014; Published 27 February 2014

Academic Editor: Claudio Borghi

Copyright (C) 2014 Dingliang Zhu et al. This is an open access article distributed under the Creative Commons Attribution License, which permits unrestricted use, distribution, and reproduction in any medium, provided the original work is properly cited.

\begin{abstract}
Objective. To evaluate the efficacy and safety of the telmisartan plus amlodipine (T/A) single-pill combination (SPC) in Asian patients with hypertension whose blood pressure (BP) was not adequately controlled on either monotherapy or on low-dose combination therapy. Patients and Methods. Data are presented from five Boehringer Ingelheim-sponsored phase 3, double-blind, 8-week, studies: two studies in nonresponders to amlodipine (data pooled for amlodipine), two studies on nonresponders to telmisartan (pooled data), and one on nonresponders to low-dose T/A SPC. Results. After 8 weeks' treatment, mean reductions from the reference baseline in diastolic BP (DBP; primary endpoint), systolic BP (SBP), and SBP, DBP goal, and response rates were higher with the T/A SPC than respective monotherapies. The T80/A5 SPC resulted in greater reductions in DBP and SBP, and higher DBP goal and response rate than the low-dose T40/A5 SPC. Peripheral edema incidence was low (amlodipine 0.5\%, telmisartan $0.0 \%$, and T/A SPC $0.7 \%$ ). Discussion and Conclusion. In Asian patients whose BP is not adequately controlled with telmisartan or amlodipine monotherapy, T/A SPC treatment results in greater BP reduction, and higher DBP and SBP goal and response rates. The safety and tolerability of the T/A SPC are comparable to those of the respective monotherapies and consistent with those reported in previous studies.
\end{abstract}

\section{Introduction}

In the Asia Pacific Cohort Studies Collaboration, up to 66\% of some subtypes of cardiovascular (CV) disease in the Asia Pacific region were attributed to hypertension [1]. High blood pressure (BP) was associated with an increased risk for CV diseases, stroke, and heart disease among the Japanese [2-4] and Chinese population [5]. The Evidence for Cardiovascular Prevention from Observational Cohorts in Japan Research Group (EPOCH-JAPAN) study reported an approximate $20 \%$ hypertension prevalence in the Japanese population [6]. Similarly, in the Chinese population, overall hypertension prevalence has been reported to be $21.5 \%$ [7], with a higher prevalence (59.4\%) reported in the elderly [8]. In Asians in general, the $\mathrm{BP}$ control rates are low, and there is a greater association between $\mathrm{BP}$ and $\mathrm{CV}$ risk [9].

Rapid and sustained BP goal achievement is important to reduce $\mathrm{CV}$ risk. At least $75 \%$ of patients with hypertension require combination therapy to achieve early $\mathrm{BP}$ goal [10], and guidelines recommend fixed-dose singlepill combinations (SPC) for their simplicity of treatment, convenience, and cost effectiveness [11, 12]. SPCs improve treatment adherence, resulting in better $\mathrm{BP}$ control and longterm CV risk reduction [13-15]. Treatment with SPCs has also resulted in significant annual cost savings [16, 17]. Significant improvement in compliance and nonsignificant beneficial trends in BP and adverse effects have been observed with SPCs compared with free drug combinations [18]. 
A renin-angiotensin system (RAS) inhibitor plus a calcium channel blocker (CCB) combination is recommended as a rational combination for hypertension treatment [11, $12,19,20]$ and is also the preferred combination in patients at high $\mathrm{CV}$ risk and those with evidence of renal disease, due to its CV and renoprotective benefits [21-23]. Telmisartan is the only angiotensin receptor blocker (ARBs) with demonstrated $\mathrm{CV}$ risk reduction similar to the angiotensinconverting enzyme (ACE) inhibitor ramipril, in patients at high $\mathrm{CV}$ risk [24] and provides superior and consistent BP reductions over 24 hours and beyond compared with other ARBs [25] and antihypertensive agents [26]. A substudy of ONTARGET (the Ongoing Telmisartan Alone and in Combination with Ramipril Global Endpoint Trial) and TRANSCEND (Telmisartan Randomized Assessment Study in ACE Intolerant Subjects with Cardiovascular Disease) that compared the tolerability of telmisartan and ramipril in Asian versus non-Asian patients showed that the advantage of better tolerability with telmisartan than ramipril was greater among Asian than non-Asian patients [27]. The telmisartan plus amlodipine (T/A) SPC (MICAMLO) is approved in Japan at the dose combinations of telmisartan $40 \mathrm{mg} /$ amlodipine $5 \mathrm{mg}$ (T40/A5) and telmisartan $80 \mathrm{mg} / \mathrm{A} 5$ (T80/A5) for the treatment of hypertension in patients not controlled on either monotherapy. The T/A SPC is approved for the treatment of hypertension as initial therapy, add-on therapy, or replacement therapy in Vietnam (doses: T40/A5 and T80/A5) and Malaysia (T40/A5, T40/amlodipine $10 \mathrm{mg}$ [A10], T80/A5, and T80/A10) and as add-on or replacement therapy in Taiwan (doses: T40/A5, T40/A10, T80/A5, snf T80/A10) and Korea (doses: T40/A5 and T80/A10 as add-on or replacement therapy; T80/A5 and T40/A10 as add-on therapy).

The objective of this analysis was to evaluate the efficacy and safety of the T/A SPC in Asian patients with hypertension whose BP was not adequately controlled on either amlodipine or telmisartan monotherapy or on low-dose combination therapy.

\section{Patients and Methods}

2.1. Studies. Five Boehringer Ingelheim-sponsored studies assessed the T/A combination in Asian patients with hypertension: two studies on nonresponders to amlodipine monotherapy (A5 nonresponder study 1 [NCT00558064], A5 nonresponder study 2 [28] [NCT01103960]), two studies on nonresponders to telmisartan monotherapy (T40 nonresponders study [NCT00550953], telmisartan T80 nonresponders study [NCT01222520]), and one study on nonresponders to low-dose T/A combination (T40/A5 nonresponders [NCT01286558]) (Table 1).

2.2. Patients. In all studies, patients included were those with essential hypertension, at least 20 years old ( $\geq 18$ years old in the A5 nonresponder study 2), and of either sex. In all studies, patients with secondary hypertension, any significant or unstable systemic disease, and previous experience of symptoms characteristic of angioedema during treatment with ACE inhibitors or ARBs and women who were pregnant, breastfeeding, or planning to become pregnant were excluded. Additional exclusion criteria at screening were treatment with four or more antihypertensive medications in the T40 nonresponder study and A5 nonresponder study 1; and three or more antihypertensive medications in the T80 nonresponder study and nonresponder to low-dose combination study. In all studies, those noncompliant with study medication during the open-label run-in period (defined as having taken $<80 \%$ or $>120 \%$ of prescribed medication, based on pill count) were also excluded. The BP inclusion and exclusion criteria are provided in Table 1.

All five studies were carried out in compliance with the protocol, the principles laid down in the Declaration of Helsinki, the International Conference on Harmonisation's Harmonised Tripartite Guidelines for Good Clinical Practice, and applicable regulatory requirements. The study protocols were reviewed by independent ethics committees or institutional review boards at each study site, and all patients provided written informed consent before entering the studies.

2.3. Study Design. All five studies were phase 3, prospective, multicenter, randomized, active control, double-dummy, double-blind, and parallel group studies. In all studies, patients who did not achieve diastolic blood pressure (DBP) goal $<90 \mathrm{~mm} \mathrm{Hg}$ ( $<80 \mathrm{~mm} \mathrm{Hg}$ in the nonresponder to lowdose combination study) with treatment during the openlabel run-in period were randomly allocated in a $1: 1$ ratio to double-blind treatment for 8 weeks. The study details are provided in Table 1.

2.4. Efficacy Assessments. In all five studies, $\mathrm{BP}$ and pulse rate were measured in the morning, approximately 24 hours after the last dose. Seated BP was measured at each visit using a standard validated and calibrated traditional, manual cuff sphygmomanometer, after the patient had rested in a seated position for approximately 5 minutes. Blood pressure was measured in the same arm, using the arm with the higher $\mathrm{BP}$ value as determined at the screening visit, and preferably by the same person at all study visits. The accuracy of BP measurements was increased by taking the mean of three consecutive measurements approximately 2 minutes apart. The seated pulse rate was measured during the 2-minute interval between the second and the third BP measurements.

2.5. Efficacy Endpoints. In all the studies, the primary endpoint was the reduction from the reference baseline in mean seated DBP at trough ( 24 hours postdosing) after 8 weeks of double-blind treatment. The reference baseline was defined as the BP value measured at the end of the open-label runin period, immediately before first dosing in the doubleblind treatment period. The secondary endpoints, which were the same for all studies, included change from reference baseline in seated trough systolic blood pressure (SBP); the proportion of patients achieving BP goal (mean seated trough $\mathrm{BP}<140 / 90 \mathrm{~mm} \mathrm{Hg}$ ); DBP goal attainment (mean seated trough DBP $<90 \mathrm{~mm} \mathrm{Hg}$ ); SBP goal attainment (mean seated trough $\mathrm{SBP}<140 \mathrm{~mm} \mathrm{Hg}$ ); the proportion of patients 


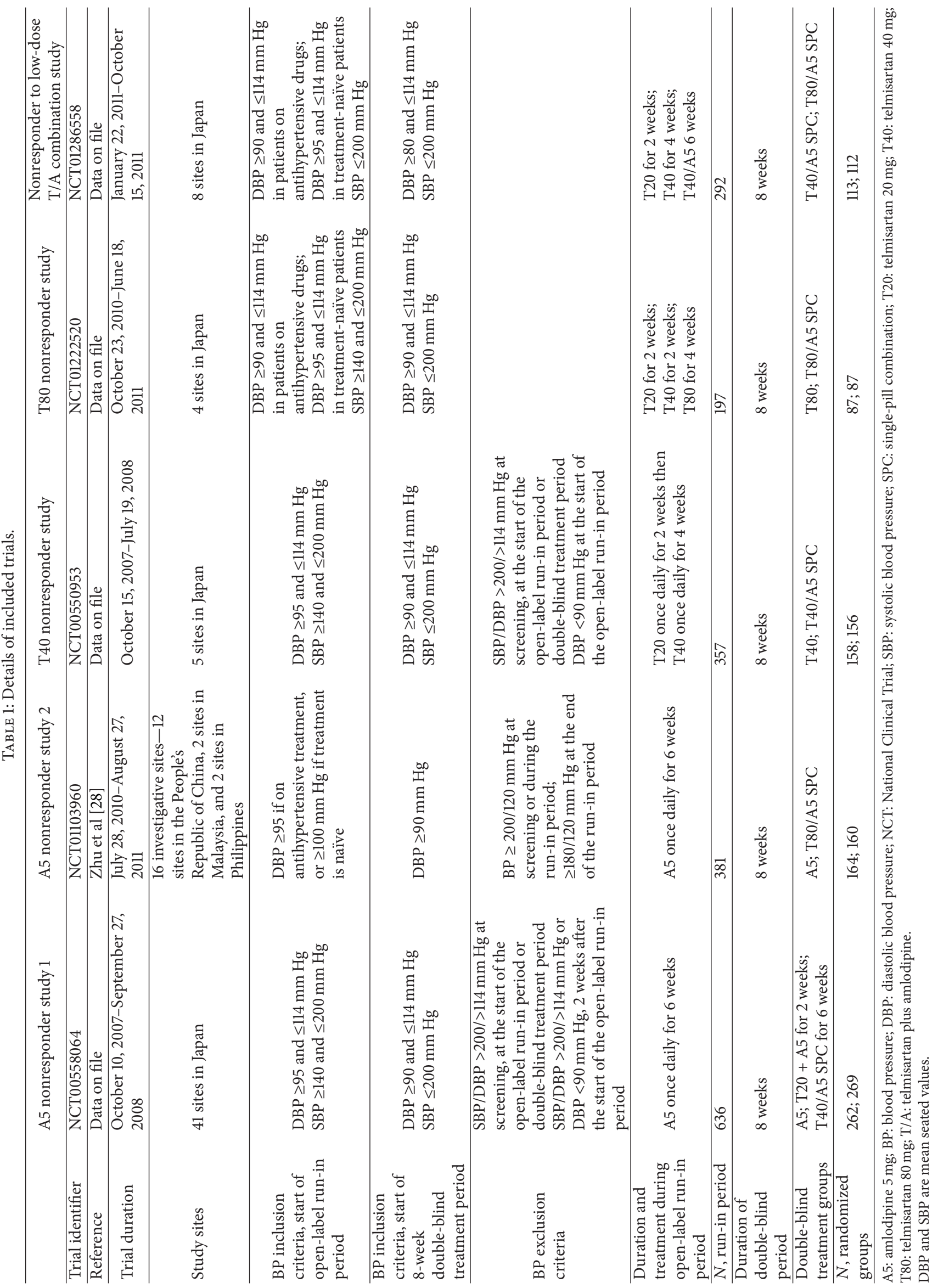


achieving DBP response (mean seated trough DBP < $90 \mathrm{~mm} \mathrm{Hg}$ or DBP reduction $\geq 10 \mathrm{~mm} \mathrm{Hg}$ ); and the proportion of patients achieving SBP response (mean seated trough $\mathrm{SBP}<140 \mathrm{~mm} \mathrm{Hg}$ or SBP reduction $\geq 20 \mathrm{~mm} \mathrm{Hg}[\geq 15 \mathrm{~mm} \mathrm{Hg}$ in A5 nonresponder study 2]). Efficacy endpoints were assessed after 4 and 8 weeks' treatment or at last through observation during the double-blind treatment period.

2.6. Safety Assessments. In all five studies, physical examination, laboratory testing, and 12-lead electrocardiogram assessment were carried out at screening, at randomization, and at the end of the study or at early withdrawal. Pulse rate and adverse events (AEs) were recorded at all visits.

2.7. Statistical Analyses. In the confirmatory analysis of all five studies, DBP and SBP reduction after 8 weeks of doubleblind treatment was analyzed using an analysis of covariance (ANCOVA) model with treatment as fixed effect, center as random effect, and reference baseline as covariate; in the A5 nonresponder study 2, country (instead of center) was included. Last trough observation carried forward was used to impute missing data for the endpoints involving seated trough BP measurements. Safety endpoints were descriptively summarized.

The statistics of this overview are purely descriptive. The full analysis set, which included all patients who took at least one dose of investigational treatment and for whom a reference baseline measurement and at least one BP measurement on randomized treatment were available, was used for demographic and baseline characteristics and efficacy analysis. The treated set, which included all randomized patients who took at least one dose of investigational treatment, was used for safety analysis. SAS version 9.3 (SAS Institute Inc., Cary, NC, USA) was used for the analyses.

Data from the five studies are presented in three groups(1) nonresponders to A5: data from the two amlodipine nonresponder studies (data pooled only for the A5 group from the two studies); (2) nonresponders to T40 or T80: data from the two telmisartan nonresponder studies; and (3) nonresponders to the T40/A5 combination: data from the one study on T40/A5 nonresponders.

\section{Results}

3.1. Patients. A total of 1542 patients were enrolled across the five studies. The demographic and baseline characteristics of the patients were similar in the three study groups. The patients' mean age was in the range of 52-57 years; most $(>60 \%)$ patients were $<60$ years of age, and most patients were men in each of the treatment subgroups included in the three study groups (Table 2). All patients were Asian, and $>84 \%$ did not have a history of diabetes. The mean duration of hypertension was 6.6-8.6 years. Mean SBP/DBP at time of randomization (reference baseline) was $>140 />95 \mathrm{~mm} \mathrm{Hg}$ in the monotherapy nonresponder trials and $>130 / 90$ in nonresponders to the low-dose combination (Table 2).

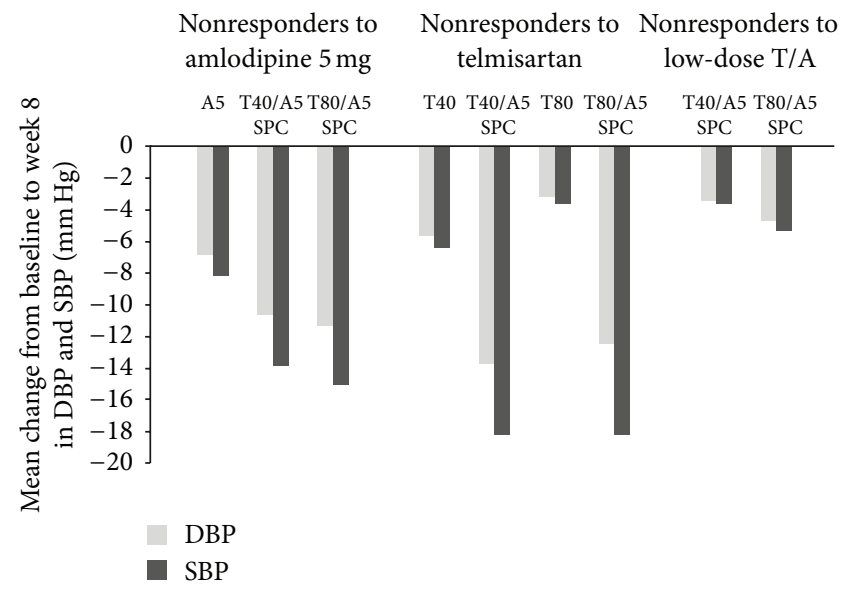

FIgURE 1: Change from baseline in DBP and SBP in the three groups after 8 weeks' treatment with T/A combination. Baseline indicates reference baseline $\mathrm{BP}$ value, which was measured immediately before first dosing in the double-blind treatment period. A5: amlodipine $5 \mathrm{mg}$; BP: blood pressure; DBP: diastolic blood pressure; SBP: systolic blood pressure; SPC: single-pill combination; T/A: telmisartan/amlodipine; T40: telmisartan $40 \mathrm{mg}$; T80: telmisartan $80 \mathrm{mg}$.

3.2. Efficacy. In the individual studies, the adjusted mean treatment difference in DBP reduction was significantly higher with the T/A SPC than with either of the monotherapies (Table 3). The T80/A5 SPC also resulted in numerically greater reductions than the low-dose combination in nonresponders to T40/A5 (Table 3). Figure 1 displays mean reductions in DBP and SBP from the reference baseline after 8 weeks of treatment for the three study groups. The advantages of the T/A SPC were consistent across age groups for the three study groups (Figures 2(a), 2(b), and 2(c)).

The BP goal attainment rates $(<140 /<90 \mathrm{~mm} \mathrm{Hg})$ at weeks 4 and 8 were higher with the T/A SPC in the nonresponders to amlodipine group and nonresponders to telmisartan group and also with the T80/A5 SPC compared with the T40/A5 SPC (Table 4). The percentage of patients achieving seated trough DBP and SBP goal and the response rates for DBP and SBP at week 8 were also higher with the T/A SPC than with the respective monotherapies (Table 4). The T80/A5 SPC resulted in higher DBP goal rates and DBP response rates at week 8 and in a higher SBP goal rate at week 4 compared with the T40/A5 combination (Table 4).

3.3. Safety. The overall incidence of AEs, discontinuation due to AEs, and incidence of peripheral edema in the individual studies are provided in Table 3. There were no deaths reported during the randomized treatment period in any of the five studies. Overall, 426 patients were exposed to A5 monotherapy, 245 patients to T40 or T80 monotherapy, and 897 patients to the T/A SPC (Table 5). The percentage of patients with any AEs was numerically lower with telmisartan and the T/A SPC than with amlodipine (Table 5). The occurrence of serious AEs was low and similar with amlodipine (angina pectoris $n=1$ and cerebellar infarction $n=1$ ) and 


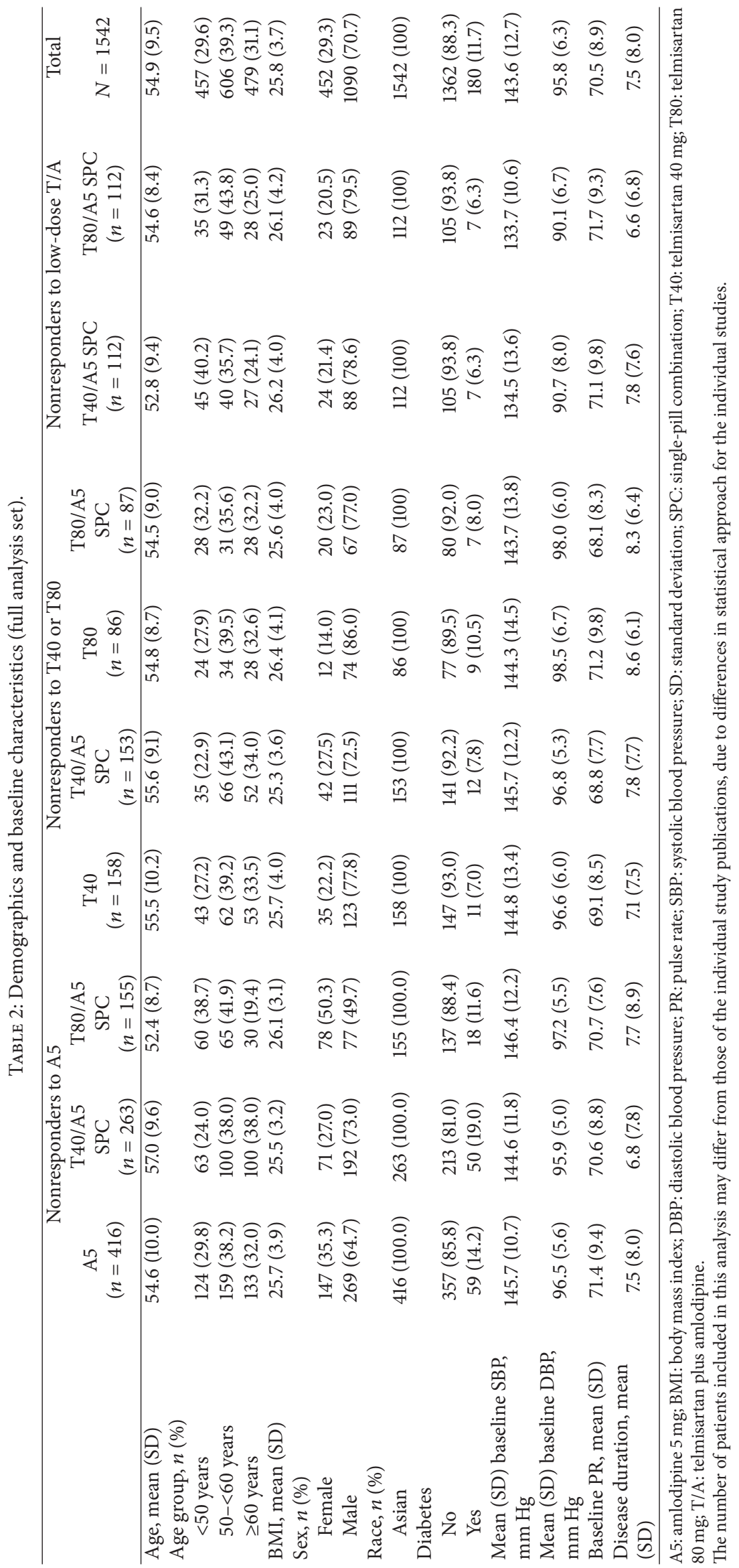




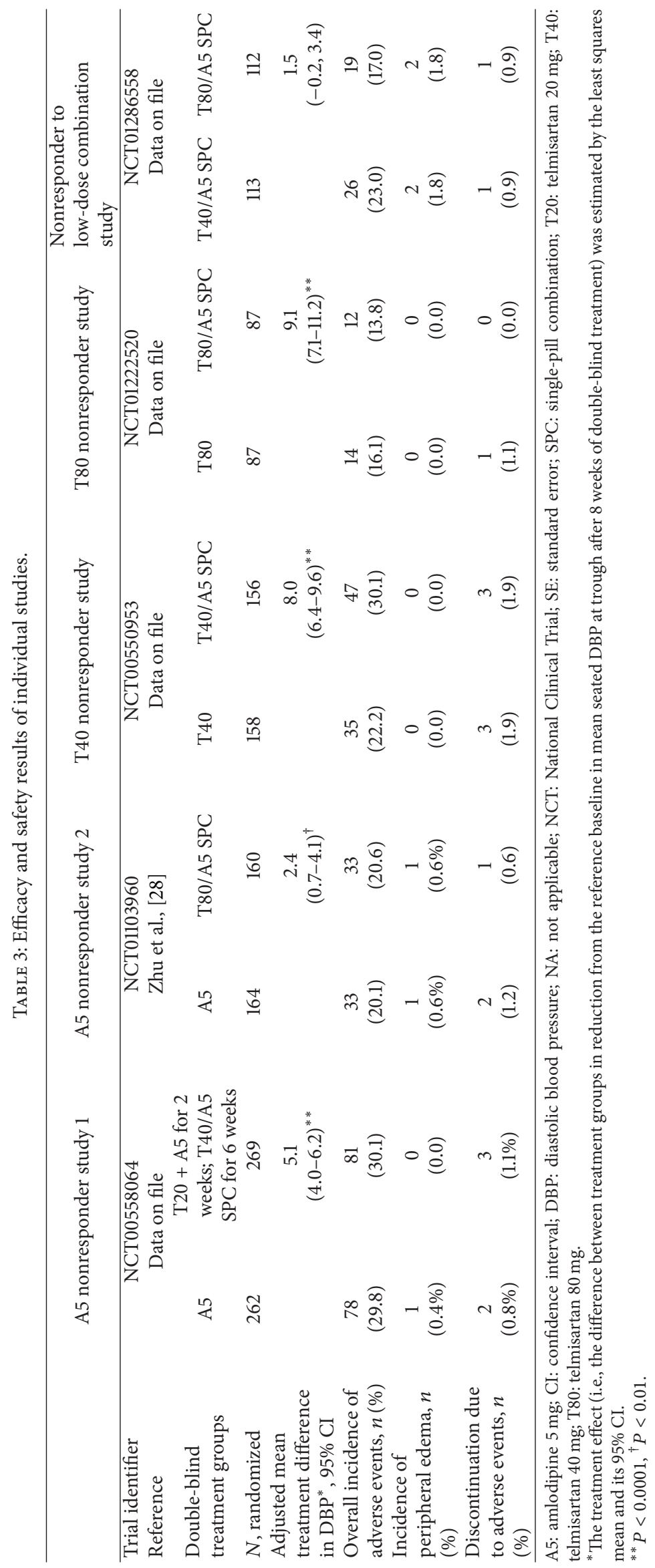




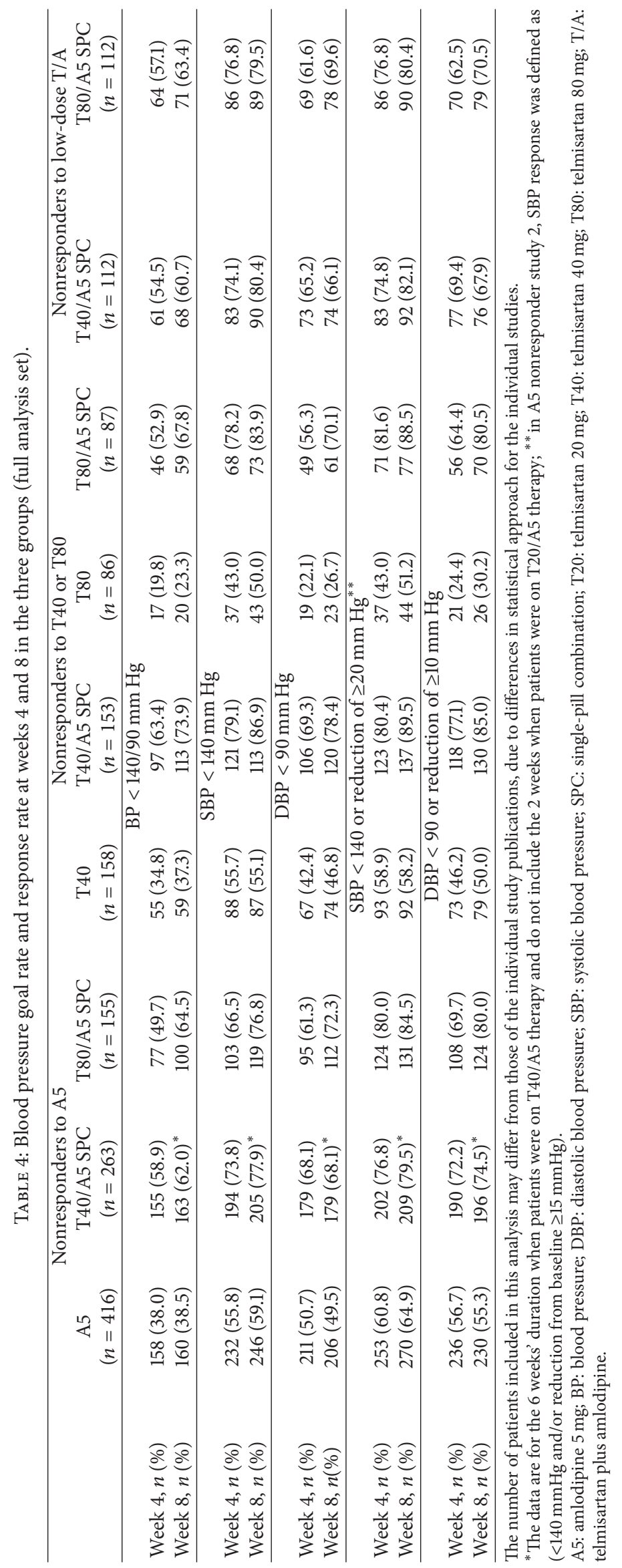




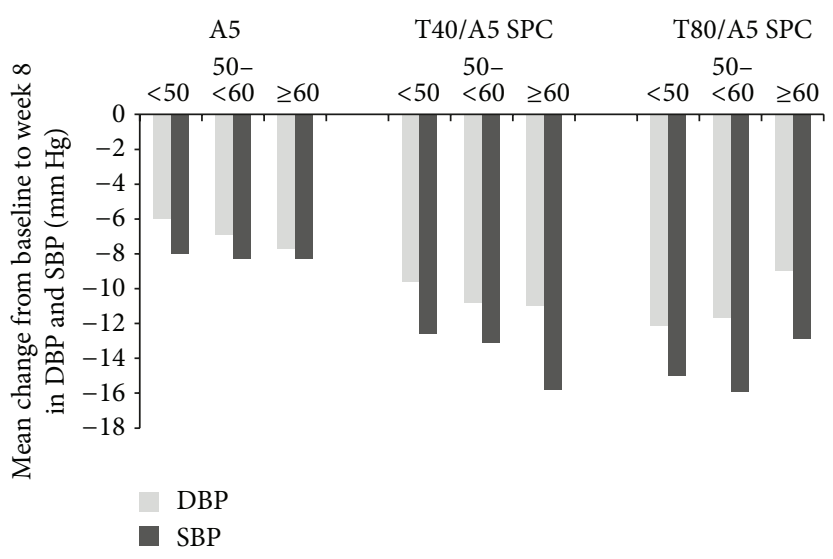

(a) Nonresponders to amlodipine $5 \mathrm{mg}$

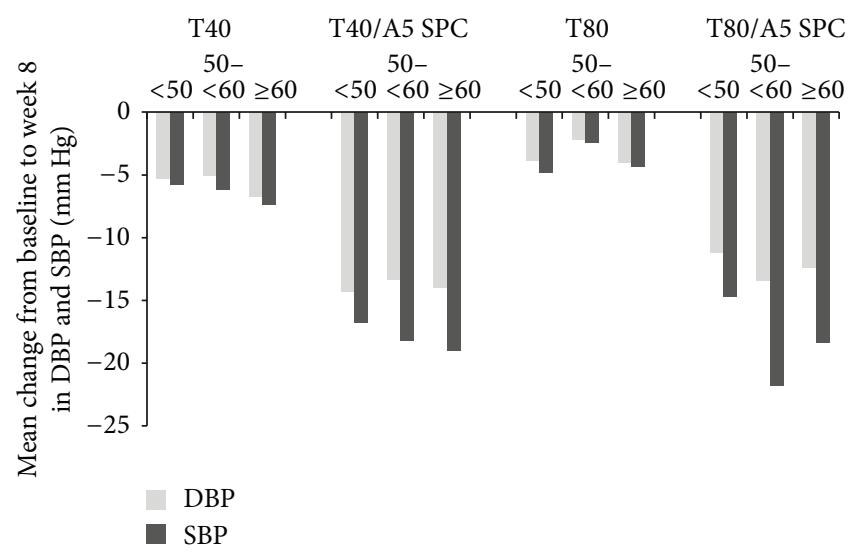

(b) Nonresponders to telmisartan

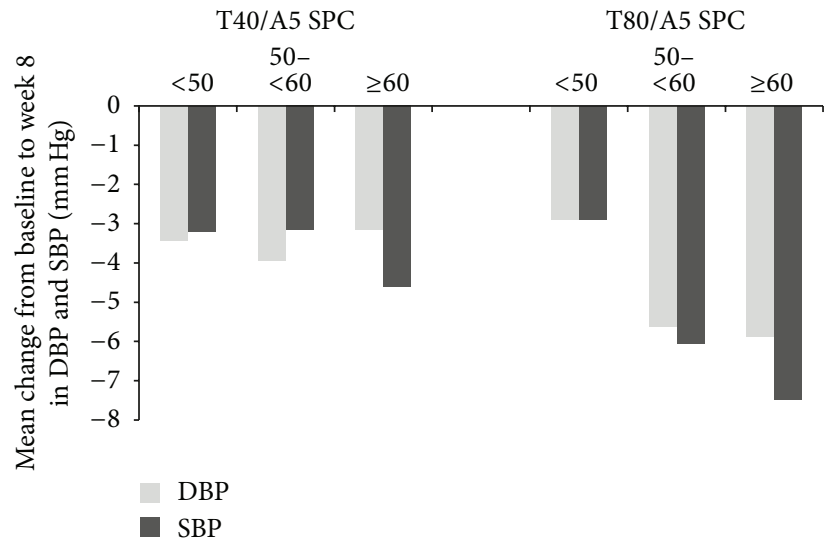

(c) Nonresponders to low-dose T/A SPC

FIGURE 2: Change from baseline in DBP and SBP by age group in the three groups after 8 weeks' treatment. Baseline indicates reference baseline BP value, which was measured immediately before first dosing in the double-blind treatment period. A5: amlodipine 5 mg; BP: blood pressure; DBP: diastolic blood pressure; SBP: systolic blood pressure; SPC: single-pill combination; T/A: telmisartan/amlodipine; T40: telmisartan $40 \mathrm{mg}$; T80: telmisartan $80 \mathrm{mg}$.

the T/A SPC (Guillain-Barré syndrome $n=1$, forearm fracture $n=1$, cerebral artery occlusion $n=1$, and clavicle fracture $n=1$ ); no serious AEs were reported with telmisartan. The incidence of Guillain-Barré syndrome, cerebellar infarction, and clavicle fracture resulted in study discontinuation. Overall, less than $2 \%$ of patients in each group discontinued the study due to AEs.

The most common AEs that occurred in more than one patient on at least one of the treatments were nasopharyngitis, gastroenteritis, dizziness, peripheral edema, bronchitis, and back pain (Table 5). Nasopharyngitis was the AE with the highest incidence and occurred in a numerically higher percentage of patients on amlodipine (8.7\%) and the T/A SPC (7.0\%) than in patients on telmisartan (1.6\%) (Table 5). Peripheral edema incidence was low and occurred in a similar percentage of patients on amlodipine $(0.5 \%)$ and the T/A SPC $(0.7 \%)$ and in no patients on telmisartan (Table 5).

\section{Discussion}

Patients with hypertension are a heterogeneous population encompassing many hypertensive phenotypes. Hence, combination therapy with pharmacologic action on two or more different physiologic sites is expected to be more effective, as it blocks the counterregulatory responses generally associated with monotherapy and its mostly single site of action [29]. Treatment with SPCs results in better BP control and long-term CV risk reduction [13, 30-32]. A prespecified substudy of the BP-lowering arm of the Anglo-Scandinavian Cardiac Outcomes Trial (ASCOT-BPLA) showed a RAS inhibitor plus a CCB to be a preferable and effective combination for South Asian patients [33].

In all the five studies reported here, the T/A SPC was more effective in lowering BP in Asian patients with hypertension whose BP was not adequately controlled with either monotherapy or low-dose combination therapy. The BP goal attainment rate and response rates at the end of 8 weeks were also higher with the T/A SPC than the respective monotherapies, and the DBP goal and response rate were higher with the T/A SPC than the low-dose T/A SPC. Similar beneficial results have been observed with the T/A SPC in other studies conducted in Asian patients. In the Cotalo study in Japanese patients whose BP was not adequately controlled with A5 monotherapy, 8 weeks' treatment with 
TABLE 5: Safety results of 8 weeks' treatment with T/A combination or the respective monotherapies.

\begin{tabular}{|c|c|c|c|}
\hline & Amlodipine monotherapy & Telmisartan monotherapy & T/A SPC therapy \\
\hline Patients treated, $n$ & 426 & 245 & 897 \\
\hline Total exposure (patient-years) & 66.6 & 31.6 & 151.8 \\
\hline Patients with any AE, $n(\%)$ & $111(26.1)$ & $49(20.0)$ & $218(24.3)$ \\
\hline Patients with drug-related AEs, $n(\%)$ & $9(2.1)$ & $3(1.2)$ & $24(2.7)$ \\
\hline Patients with SAE, $n(\%)$ & $2(0.5)$ & $0(0)$ & $4(0.4)$ \\
\hline Patients with AEs leading to discontinuation, $n(\%)$ & $4(0.9)$ & $4(1.6)$ & $9(1.0)$ \\
\hline \multicolumn{4}{|c|}{ Most common AEs that occurred in more than one patient on at least one of the treatments, $n(\%)$} \\
\hline Nasopharyngitis & $37(8.7)$ & $4(1.6)$ & $63(7.0)$ \\
\hline Gastroenteritis & $4(0.9)$ & $5(2.0)$ & $4(0.4)$ \\
\hline Dizziness & $4(0.9)$ & $0(0)$ & $8(0.9)$ \\
\hline Peripheral edema & $2(0.5)$ & $0(0)$ & $6(0.7)$ \\
\hline Bronchitis & $2(0.5)$ & $0(0)$ & $5(0.6)$ \\
\hline Back pain & $2(0.5)$ & $1(0.4)$ & $3(0.3)$ \\
\hline
\end{tabular}

AE: adverse event; SAE: serious adverse event; SPC: single-pill combination; T/A: telmisartan plus amlodipine.

The number of patients included in this analysis may differ from those of the individual study publications, due to differences in statistical approach for the individual studies.

T40/A5 SPC significantly decreased the 24-hour mean and clinical BP, independent of administration time; results were similar between patients with or without metabolic syndrome [34]. In a prospective open-label study on Japanese patients with hypertension with BP uncontrolled on treatment with valsartan $80 \mathrm{mg} / \mathrm{A} 5$ or candesartan $8 \mathrm{mg} / \mathrm{A} 5$, switching to T40/A5 treatment resulted in a significant reduction in both mean clinic SBP and DBP at 4, 8, and 12 weeks, compared with valsartan $80 \mathrm{mg} / \mathrm{A} 5$ treatment suggesting more favorable CV outcomes with the T40/A5 combination [35]. Similar benefits were seen with T40/A5 in the subgroup of elderly patients; in addition, T40/A5 was found to significantly increase serum adiponectin levels, suggesting beneficial cardiometabolic effects in the elderly [36]. In a multicenter, open-label clinical trial in Chinese high-risk hypertensive patients with at least one $\mathrm{CV}$ risk factor, 96-week treatment with $\mathrm{T} / \mathrm{A}$ was efficacious in reducing $\mathrm{BP}$ levels with acceptable goal rates and was well tolerated [37].

The results reported here are consistent with the results from international studies conducted in patients with mildto-moderate or severe hypertension not controlled on amlodipine monotherapy [38-41] and suggest similar benefits with the T/A combination in Asian patients. In international studies, the T/A combination compared with the respective monotherapies has also been shown to provide superior 24-hour BP lowering in patients with mild-tomoderate hypertension [42] and significantly greater BP reductions and higher $\mathrm{BP}$ goal and response rates in patients with severe hypertension [43]. Planned and post hoc pooled analysis of data from clinical trials have shown the T/A SPC to be efficacious and well tolerated in hypertensive patients with added risk factors, including obesity, diabetes, metabolic syndrome, renal impairment, and elevated SBP [44-46].

Overall, double-blind treatment for 8 weeks with either monotherapy or with a T/A SPC was well tolerated in the five included studies. The safety and tolerability data obtained after 8 weeks of double-blind, randomized treatment were consistent with the known safety and tolerability of telmisartan and amlodipine or the T/A SPC, and no clinically important differences were noted. In previous studies, T/A SPC treatment was associated with a lower incidence of peripheral edema than with amlodipine monotherapy [3841]. In this analysis of studies on Asian patients, the incidence of peripheral edema was low and was reported in less than $1 \%$ of patients on amlodipine monotherapy and the T/A SPC and in no patients receiving telmisartan monotherapy. The overall lower incidence of edema observed in this study could be related to the differences between ethnic groups in risks for certain AEs.

The studies reported here included only Japanese, Chinese, Malaysian, and Philippine patients, which may limit the applicability of the findings to patients from other Asian countries. Also, the controlled nature of the studies limits generalization of the results to those categories of patients who were excluded from these studies.

\section{Conclusions}

In Asian patients whose BP is not adequately controlled with telmisartan or amlodipine monotherapy, 8 weeks' treatment with a T/A combination results in greater BP reduction and higher DBP and SBP goal and response rates. In Asian patients whose $\mathrm{BP}$ is not adequately controlled with the T40/A5 SPC, 8 weeks' treatment with the T80/A5 SPC results in greater $\mathrm{BP}$ reduction and $\mathrm{DBP}$ goal and response rates. The safety and tolerability profile of the T/A SPC is comparable to that of the respective monotherapies and consistent with that reported in previous studies.

\section{Disclosure}

Mr. Yagi is an employee of Nippon Boehringer Ingelheim Co., Ltd, Japan. Dr. Schumacher is an employee of Boehringer Ingelheim Pharma GmbH \& Co. KG, Germany. 


\section{Conflict of Interests}

Dr. Zhu and Dr. Gao declare no potential conflict of interests.

\section{Acknowledgments}

The authors were fully responsible for all content and editorial decisions, were involved at all stages of manuscript development, and have approved the final version. Medical writing assistance, supported financially by Boehringer Ingelheim Pharma GmbH \& Co. KG, was provided by Lakshmi Venkatraman, PhD, of PAREXEL during the preparation of this paper. The authors meet criteria for authorship as recommended by the International Committee of Medical Journal Editors (ICMJE) and received no compensation related to the development of the paper. The authors thank the study participants, without whom the studies would not have been possible, and the following investigators for their participation from China: Chen Jun Zhu; Dai Qiuyan; Dong Yugang; Gu Shuiming; Hu Dayi; Li Hongwei; Li Huimin; Li Zhanquan; Luo Ming; Zhang Yu; Zhao Shui Ping; Zhu Dingliang; Japan: Akira Hakoshima; Akira Ohishi; Akiyoshi Amino; Chiaki Noguchi; Fumitaka Shinojima; Hajime Fujita; Haruna Azuma; Hideo Takahashi; Hidetaka Hougaku; Hirokazu Kitada; Hiromu Tej; Hiroshi Kazuma; Hiroshi Mikami; Hiroyasu Konishi; Isao Hataoka; Katsuhiro Matsuda; Kenji Kobayashi; Koichi Kimura; Kouji Sanda; Masahiro Yamamoto; Masakuni Suematsu; Narumi Sugimori; Osamu Kinoshita; Osamu Matsuoka; Satoshi Inoue; Sayuri Hirota; Seiji Eto; Shigenao Kan; Shintaro Shokoku; Shunya Sato; Tadashi Fujikawa; Takayuki Azuma; Takeo Higashikata; Takuro Ichikawa; Teruaki Mita; Tetsuro Hiraoka; Toshihiko Shiraiwa; Toshiki Fukui; Toyohisa Eguchi; Yasuhiro Nakamura; Yasushi Amada; Yasushi Wakida; Yasuo Tanno; Yoji Takatsuka; Yoshinori Keira; Yuichiro Nakamura; Yutaka Kadono; Yuriko Tarukawa; Malaysia, Azhari Rosman; Jeyaindran Sinnadurai; Philippines: Marcelito Durante; Philip U. Chua.

\section{References}

[1] A. L. Martiniuk, C. M. Lee, C. M. M. Lawes et al., "Hypertension: its prevalence and population-attributable fraction for mortality from cardiovascular disease in the Asia-Pacific region," Journal of Hypertension, vol. 25, no. 1, pp. 73-79, 2007.

[2] H. Ueshima, O. Iimura, M. Iida, A. Okayama, K. Sawai, and M. Minowa, "Impact of elevated blood pressure on mortality from all causes, cardiovascular diseases, heart disease and stroke among Japanese: 14 year follow-up of randomly selected population from Japanese-nippon data 80," Journal of Human Hypertension, vol. 17, no. 12, pp. 851-857, 2003.

[3] K. Miura, "Epidemiology and prevention of hypertension in Japanese: how could Japan get longevity?" EPMA Journal, vol. 2, no. 1, pp. 59-64, 2011.

[4] A. Okayama, T. Kadowaki, T. Okamura, T. Hayakawa, and H. Ueshima, "Age-specific effects of systolic and diastolic blood pressures on mortality due to cardiovascular diseases among Japanese men (NIPPON DATA80)," Journal of Hypertension, vol. 24 , no. 3, pp. 459-462, 2006.
[5] D. Gu, T. N. Kelly, X. Wu et al., "Blood pressure and risk of cardiovascular disease in chinese men and women," The American Journal of Hypertension, vol. 21, no. 3, pp. 265-272, 2008.

[6] Y. Murakami, A. Hozawa, T. Okamura, and H. Ueshima, "Relation of blood pressure and all-cause mortality in 180, 000 japanese participants pooled analysis of 13 cohort studies," Hypertension, vol. 51, no. 6, pp. 1483-1491, 2008.

[7] Y. Q. Ma, W. H. Mei, P. Yin, X. H. Yang, S. K. Rastegar, and J. D. Yan, "Prevalence of hypertension in Chinese cities: a metaanalysis of published studies," PLoS ONE, vol. 8, no. 3, Article ID e58302, 2013.

[8] C. S. Sheng, M. Liu, Y. Y. Kang et al., "Prevalence, awareness, treatment and control of hypertension in elderly Chinese," Hypertension Research, vol. 36, no. 9, pp. 824-828, 2013.

[9] S. E. Kjeldsen, F. H. Messerli, C. E. Chiang, P. A. Meredith, and L. Liu, "Are fixed-dose combination antihypertensives suitable as first-line therapy?" Current Medical Research and Opinion, vol. 28, no. 10, pp. 1685-1697, 2012.

[10] A. H. Gradman, J. N. Basile, B. L. Carter, and G. L. Bakris, "Combination therapy in hypertension," Journal of the American Society of Hypertension, vol. 4, no. 2, pp. 90-98, 2010.

[11] A. V. Chobanian, G. L. Bakris, H. R. Black et al., "Seventh report of the joint national committee on prevention, detection, evaluation, and treatment of high blood pressure," Hypertension, vol. 42, no. 6, pp. 1206-1252, 2003.

[12] G. Mancia, R. Fagard, K. Narkiewicz et al., "ESH/ESC Guidelines for the management of arterial hypertension: the Task Force for the management of arterial hypertension of the European Society of Hypertension (ESH) and of the European Society of Cardiology (ESC)," European Heart Journal, vol. 34, no. 28, pp. 2159-2219, 2013.

[13] S. Bangalore and L. Ley, "Improving treatment adherence to antihypertensive therapy: the role of single-pill combinations," Expert Opinion on Pharmacotherapy, vol. 13, no. 3, pp. 345-355, 2012.

[14] B. Sherrill, M. Halpern, S. Khan, J. Zhang, and S. Panjabi, "Single-pill vs free-equivalent combination therapies for hypertension: a meta-analysis of health care costs and adherence," Journal of Clinical Hypertension, vol. 13, no. 12, pp. 898-909, 2011.

[15] S. Bangalore, G. Kamalakkannan, S. Parkar, and F. H. Messerli, "Fixed-dose combinations improve medication compliance: a meta-analysis," The American Journal of Medicine, vol. 120, no. 8, pp. 713-719, 2007.

[16] M. Akazawa and K. Fukuoka, "Economic impact of switching to fixed-dose combination therapy for Japanese hypertensive patients: a retrospective cost analysis," BMC Health Services Research, vol. 13, p. 124, 2013.

[17] G. Hess, J. Hill, H. Lau, H. Dastani, and P. Chaudhari, "Medication utilization patterns and hypertension-related expenditures among patients who were switched from fixed-dose to freecombination antihypertensive therapy," $P$ and $T$, vol. 33, no. 11, pp. 652-666, 2008.

[18] A. K. Gupta, S. Arshad, and N. R. Poulter, "Compliance, safety, and effectiveness of fixed-dose combinations of antihypertensive agents: a meta-analysis," Hypertension, vol. 55, no. 2, pp. 399-407, 2010.

[19] G. Mancia, S. Laurent, E. Agabiti-Rosei et al., "Reappraisal of European guidelines on hypertension management: a European Society of Hypertension Task Force document," Journal of Hypertension, vol. 27, no. 11, pp. 2121-2158, 2009. 
[20] T. Ogihara, K. Kikuchi, H. Matsuoka et al., "The Japanese Society of Hypertension Guidelines for the Management of Hypertension (JSH 2009)," Hypertension Research, vol. 32, no. 1, pp. 3-107, 2009.

[21] J. M. Neutel, "Treatment algorithms for hypertension: a practical approach," Clinical Practice, vol. 10, no. 3, pp. 333-344, 2013.

[22] A. F. Rubio-Guerra, D. Castro-Serna, C. I. Barrera, and L. M. Ramos-Brizuela, "Current concepts in combination therapy for the treatment of hypertension: combined calcium channel blockers and RAAS inhibitors," Integrated Blood Pressure Control, vol. 2, pp. 55-62, 2009.

[23] S. G. Mallat, "Which is the preferred angiotensin II receptor blocker-based combination therapy for blood pressure control in hypertensive patients with diabetic and non-diabetic renal impairment?" Cardiovascular Diabetology, vol. 11, p. 32, 2012.

[24] S. Yusuf, K. K. Teo, J. Pogue et al., "Telmisartan, ramipril, or both in patients at high risk for vascular events," The New England Journal of Medicine, vol. 358, no. 15, pp. 1547-1559, 2008.

[25] M. Burnier, "Telmisartan: a different angiotensin II receptor blocker protecting a different population?" Journal of International Medical Research, vol. 37, no. 6, pp. 1662-1679, 2009.

[26] D. Galzerano, C. Capogrosso, S. di Michele et al., "New standards in hypertension and cardiovascular risk management: focus on telmisartan," Vascular Health and Risk Management, vol. 6, no. 1, pp. 113-133, 2010.

[27] A. L. Dans, K. Teo, P. Gao et al., "In a subgroup of highrisk asians, telmisartan was non-inferior to ramipril and better tolerated in the prevention of cardiovascular events," PLoS ONE, vol. 5, no. 12, Article ID e13694, 2010.

[28] D. Zhu, P. Gao, W. Holtbruegge, and C. Huang, "A randomized, double-blind study to evaluate the efficacy and safety of a singlepill combination of telmisartan $80 \mathrm{mg} /$ amlodipine $5 \mathrm{mg}$ versus amlodipine $5 \mathrm{mg}$ in hypertensive Asian patients," Journal of International Medical Research, vol. 42, no. 1, pp. 52-66, 2014.

[29] P. S. Sever and F. H. Messerli, "Hypertension management 2011: optimal combination therapy," European Heart Journal, vol. 32, no. 20, pp. 2499-2506, 2011.

[30] B. M. Egan, D. Bandyopadhyay, S. R. Shaftman, C. S. Wagner, Y. Zhao, and K. S. Yu-Isenberg, "Initial monotherapy and combination therapy and hypertension control the first year," Hypertension, vol. 59, no. 6, pp. 1124-1131, 2012.

[31] A. H. Gradman, H. Parise, P. Lefebvre, H. Falvey, M. H. Lafeuille, and M. S. Duh, "Initial combination therapy reduces the risk of cardiovascular events in hypertensive patients: a matched cohort study," Hypertension, vol. 61, no. 2, pp. 309-318, 2013.

[32] G. Corrao, F. Nicotra, A. Parodi et al., "Cardiovascular protection by initial and subsequent combination of antihypertensive drugs in daily life practice," Hypertension, vol. 58, no. 4, pp. 566$572,2011$.

[33] A. K. Gupta, N. R. Poulter, J. Dobson et al., "Ethnic differences in blood pressure response to first and second-line antihypertensive therapies in patients randomized in the ASCOT trial," The American Journal of Hypertension, vol. 23, no. 9, pp. 1023-1030, 2010.

[34] M. Ohishi, T. Kawai, N. Hayashi et al., "Effect of tablets with a combination of telmisartan and amlodipine on patients with hypertension: the Cotalo study," Hypertension Research, vol. 36, no. 7, pp. 620-626, 2013.

[35] H. Bekki, K. Yamamoto, M. Sone et al., "Efficacy of combination therapy with telmisartan plus amlodipine in patients with poorly controlled hypertension," Oxidative Medicine and Cellular Longevity, vol. 3, no. 5, pp. 342-346, 2010.

[36] H. Bekki, K. Yamamoto, M. Sone et al., "Beneficial cardiometabolic actions of telmisartan plus amlodipine therapy in elderly patients with poorly controlled hypertension," Clinical Cardiology, vol. 34, no. 4, pp. 261-265, 2011.

[37] L. Ma, W. Wang, Y. Zhao et al., "Combination of amlodipine plus angiotensin receptor blocker or diuretics in high-risk hypertensive patients: a 96-week efficacy and safety study," The American Journal of Cardiovascular Drugs, vol. 12, no. 2, pp. 137142, 2012.

[38] T. W. Littlejohn III, C. R. Majul, R. Olvera et al., "Results of treatment with telmisartan-amlodipine in hypertensive patients," The Journal of Clinical Hypertension, vol. 11, no. 4, pp. 207-213, 2009.

[39] T. W. Littlejohn III, C. R. Majul, R. Olvera et al., “Telmisartan plus amlodipine in patients withmoderate or severe hypertension: results from a subgroup analysis of a randomized, placebocontrolled, parallel-group, $4 \times 4$ factorial study," Postgraduate Medicine, vol. 121, no. 2, pp. 5-14, 2009.

[40] S. Neldam, M. Lang, and R. Jones, "Telmisartan and amlodipine single-pill combinations vs amlodipine monotherapy for superior blood pressure lowering and improved tolerability in patients with uncontrolled hypertension: results of the TEAMSTA-5 study," Journal of Clinical Hypertension, vol. 13, no. 7, pp. 459-466, 2011.

[41] S. Neldam, C. Edwards, and R. Jones, "Switching patients with uncontrolled hypertension on amlodipine $10 \mathrm{mg}$ to single-pill combinations of telmisartan and amlodipine: results of the TEAMSTA-10 study," Current Medical Research and Opinion, vol. 27, no. 11, pp. 2145-2153, 2011.

[42] W. B. White, T. W. Littlejohn, C. R. Majul et al., "Effects of telmisartan and amlodipine in combination on ambulatory blood pressure in stages 1-2 hypertension," Blood Pressure Monitoring, vol. 15, no. 4, pp. 205-212, 2010.

[43] J. M. Neutel, G. Mancia, H. R. Black et al., "Single-pill combination of telmisartan/amlodipine in patients with severe hypertension: results from the TEAMSTA severe HTN study," The Journal of Clinical Hypertension, vol. 14, no. 4, pp. 206-215, 2012.

[44] R. M. Guthrie, B. Dahlöf, K. A. Jamerson et al., "Efficacy and tolerability of telmisartan plus amlodipine in added-risk hypertensive patients," Current Medical Research and Opinion, vol. 27, no. 10, pp. 1995-2008, 2011.

[45] L. Ley and H. Schumacher, "Telmisartan plus amlodipine single-pill combination for the management of hypertensive patients with a metabolic risk profile (added-risk patients)," Current Medical Research and Opinion, vol. 29, no. 1, pp. 41-53, 2013.

[46] A. M. Sharma, G. Bakris, J. M. Neutel et al., "Single-pill combination of telmisartan/Amlodipine versus amlodipine monotherapy in diabetic hypertensive patients: an 8-week randomized, parallel-group, double-blind trial," Clinical Therapeutics, vol. 34, no. 3, pp. 537-551, 2012. 


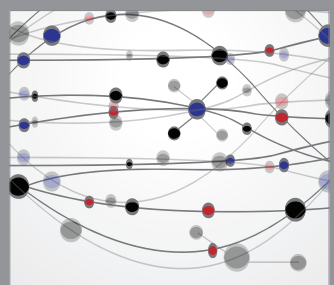

The Scientific World Journal
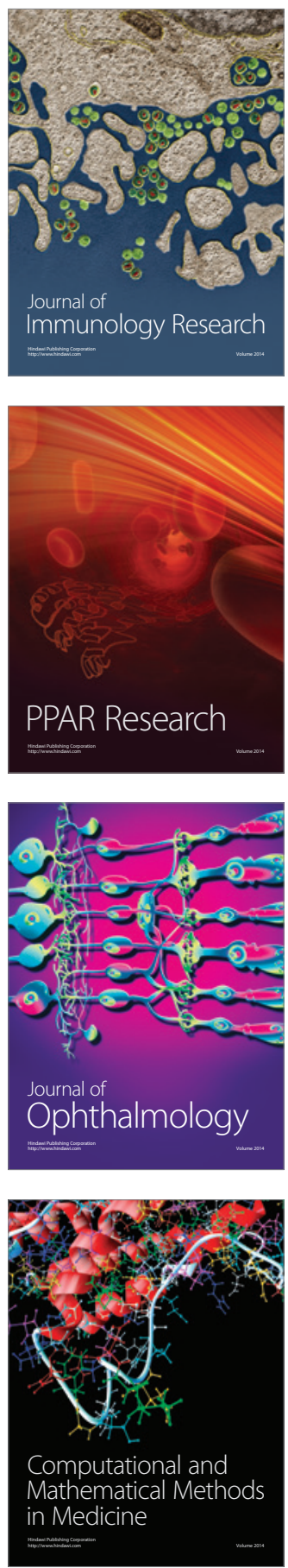

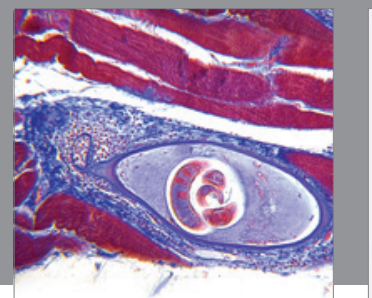

Gastroenterology

Research and Practice
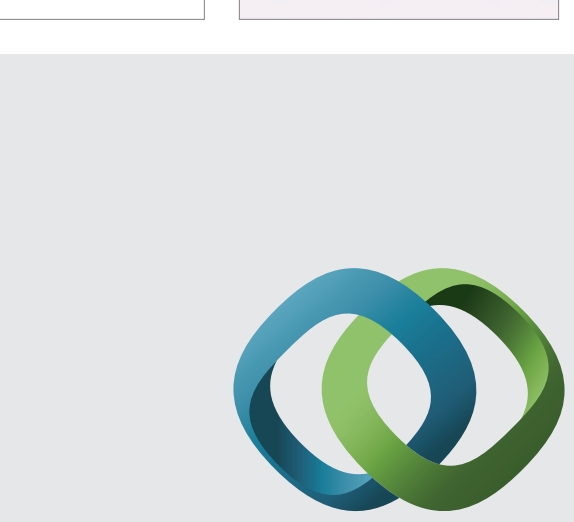

\section{Hindawi}

Submit your manuscripts at

http://www.hindawi.com
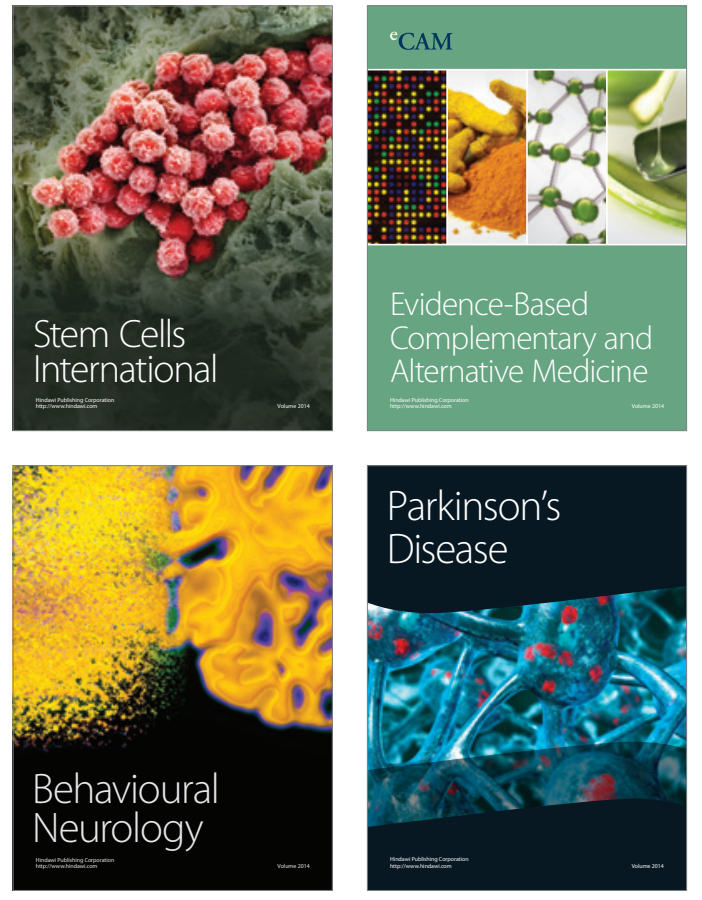
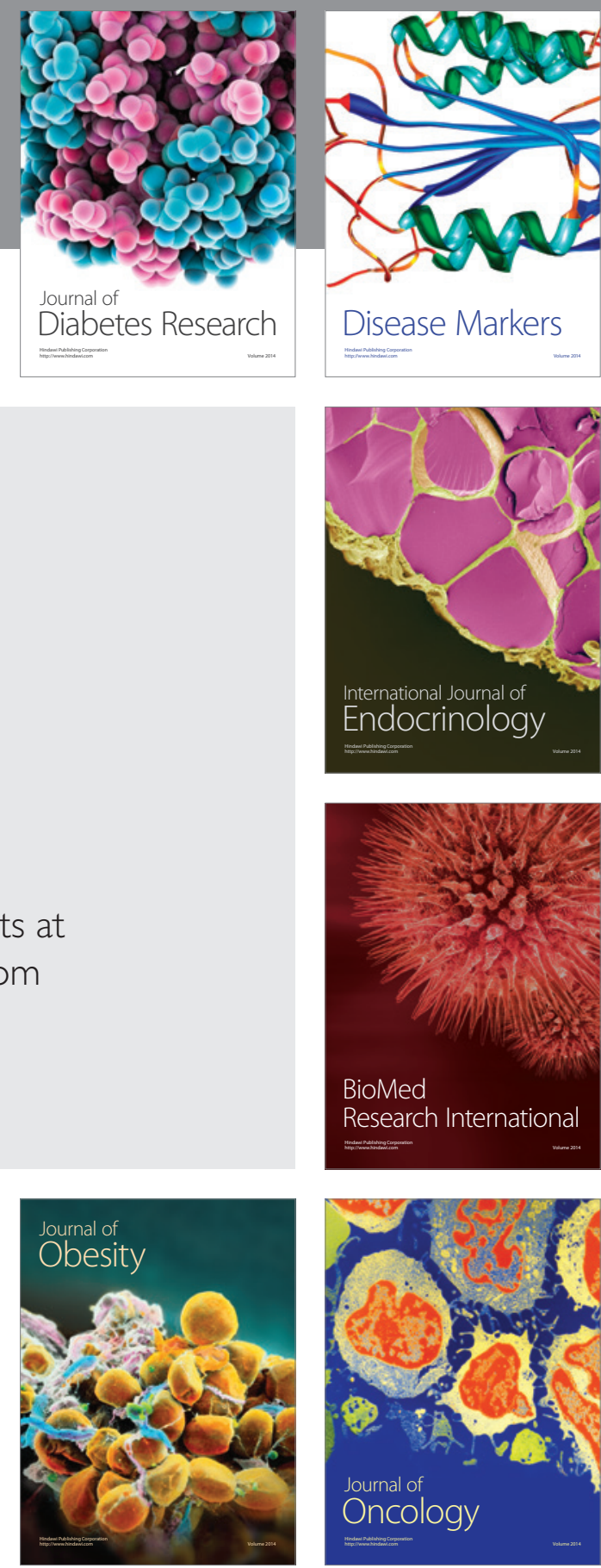

Disease Markers
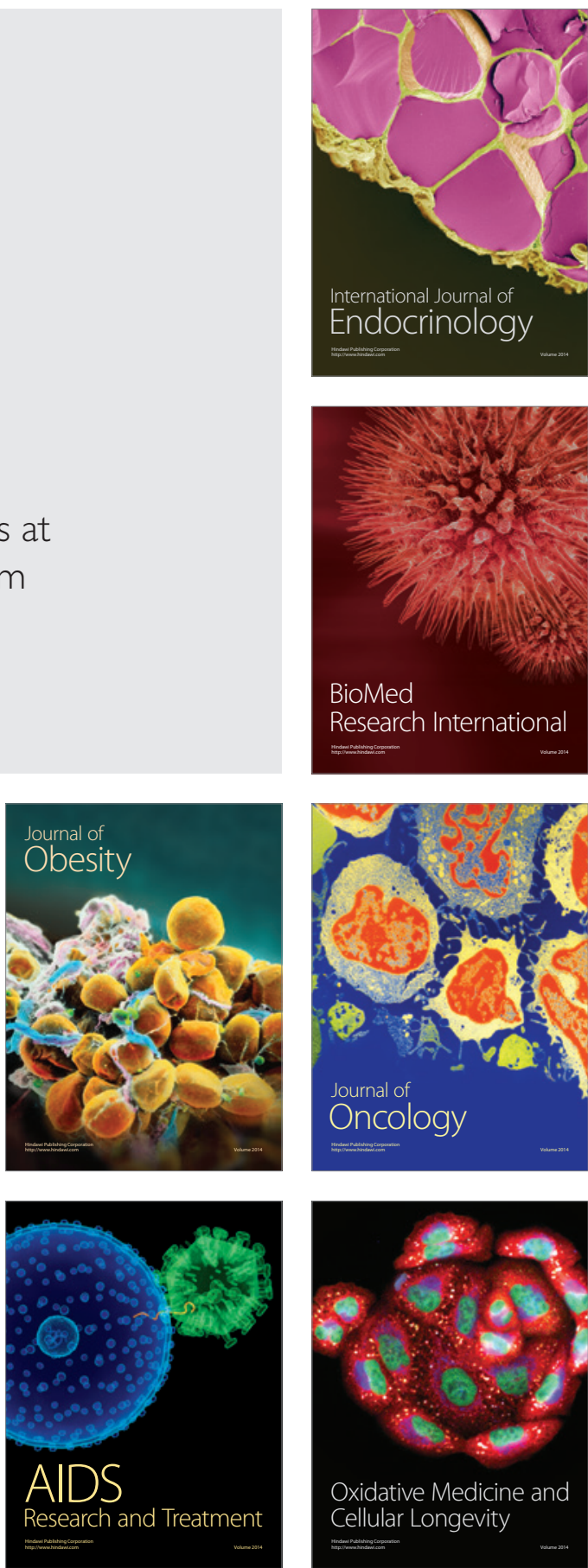